\title{
SUPERCONVERGENCE OF THE LOCAL DISCONTINUOUS GALERKIN METHOD FOR ELLIPTIC PROBLEMS ON CARTESIAN GRIDS
}

\author{
BERNARDO COCKBURN $*$, GUIDO KANSCHAT ${ }^{\dagger}$, ILARIA PERUGIA $\ddagger$, AND DOMINIK \\ SCHÖTZAU §
}

April 27, 2000

\begin{abstract}
In this paper, we present a super-convergence result for the Local Discontinuous Galerkin method for a model elliptic problem on Cartesian grids. We identify a special numerical flux for which the $\mathrm{L}^{2}$-norm of the gradient and the $\mathrm{L}^{2}$-norm of the potential are of order $k+1 / 2$ and $k+1$, respectively, when tensor product polynomials of degree at most $k$ are used; for arbitrary meshes, this special LDG method gives only the orders of convergence of $k$ and $k+1 / 2$, respectively. We present a series of numerical examples which establish the sharpness of our theoretical results.
\end{abstract}

Key words. Finite elements, discontinuous Galerkin methods, super-convergence, elliptic problems, Cartesian grids.

AMS subject classifications. Primary 65 N 30.

1. Introduction. In this paper, we derive a priori error estimates of the Local Discontinuous Galerkin (LDG) method on Cartesian grids for the following classical model elliptic problem:

$$
\begin{aligned}
-\Delta u & =f & & \text { in } \Omega, \\
u & =g_{\mathcal{D}} & & \text { on } \Gamma_{\mathcal{D}}, \\
\frac{\partial u}{\partial n} & =\boldsymbol{g}_{\mathcal{N}} \cdot \boldsymbol{n} & & \text { on } \Gamma_{\mathcal{N}},
\end{aligned}
$$

where $\Omega$ is a bounded domain of $\mathbb{R}^{d}$ and $\boldsymbol{n}$ is the outward unit normal to its boundary $\Gamma=\bar{\Gamma}_{\mathcal{D}} \cup \bar{\Gamma}_{\mathcal{N}} ;$ we assume that the $(d-1)$-measure of $\Gamma_{\mathcal{D}}$ is non-zero.

Recently, Castillo, Cockburn, Perugia and Schötzau [3] obtained the first a priori error analysis of the LDG method for purely elliptic problems. Meshes consisting of elements of various shapes and with hanging nodes were considered and general

${ }^{*}$ School of Mathematics, University of Minnesota, Vincent Hall, Minneapolis, MN 55455 (cockburn@math.umn.edu). Supported in part by the National Science Foundation (Grant DMS9807491) and by the University of Minnesota Supercomputing Institute.

$\dagger$ Institut für Angewandte Mathematik, Universität Heidelberg, INF 293/294, 69120 Heidelberg, Germany (guido.kanschat@na-net.ornl.gov). This work was supported in part by the ARO DAAG55-98-1-0335 and by the University of Minnesota Supercomputing Institute. It was carried out when the author was a Visiting Professor at the School of Mathematics, University of Minnesota.

$\ddagger$ Dipartimento di Matematica, Università di Pavia, Via Ferrata 1, 27100 Pavia, Italy (perugia@dimat.unipv.it). Supported in part by the Consiglio Nazionale delle Ricerche. This work was carried out when the author was a Visiting Professor at the School of Mathematics, University of Minnesota.

$\S$ School of Mathematics, University of Minnesota, Vincent Hall, Minneapolis, MN 55455 (schoetza@math.umn.edu). Supported by the Swiss National Science Foundation (Schweizerischer Nationalfonds). 
numerical fluxes were studied. It was shown that, for very smooth solutions, the orders of convergence of the $\mathrm{L}^{2}$-norms of the errors in $\nabla u$ and in $u$ are $k$ and $k+1 / 2$, respectively when polynomials of degree at most $k$ are used. On the other hand, Castillo [2] and Castillo, Cockburn, Schötzau and Schwab [4] proved that, for onespace dimension transient convection-diffusion problems, the order of convergence of the error in the energy norm is optimal, that is, $k+1$, provided that the so-called numerical fluxes are suitably chosen. In this paper, we extend these results to the LDG method on Cartesian grids for the multi-dimensional elliptic model problem (1.1); we show that the orders of convergence in the $\mathrm{L}^{2}$-norm of the error in $\nabla u$ and $u$ are $k+1 / 2$ and $k+1$, respectively, when tensor product polynomials of degree at least $k$ are used. Our proof of this super-convergence result is a modification of the analysis carried out in [3]; it takes advantage of the Cartesian structure of the grid and makes use of a key idea introduced by LeSaint and Raviart [10] in their study of the original DG method for steady-state linear transport.

Since our analysis is a special modification of that of [3], in order to avoid unnecessary repetitions, we refer the reader to [3] for a more detailed description of the framework of our error analysis. The organization of this paper is as follows. In Section 2, we briefly display the LDG method in compact form, introduce the special numerical flux on Cartesian grids and present and discuss our main result. In Section 3, the detailed proofs are given and in Section 4, we present several numerical experiments showing the optimality of our theoretical results. We end in Section 5 with some concluding remarks.

2. The main results. In this section we recall the formulation of the LDG method and identify the special numerical flux we are going to investigate on Cartesian grids. Then we state and discuss our main results. As pointed out in the introduction, we refer to [3] for more details concerning the formulation of the LDG method.

2.1. The LDG method. We assume that the problem domain $\Omega$ can be covered by a Cartesian grid. To define the LDG method, we rewrite our elliptic model problem (1.1) as the following system of first-order equations:

$$
\begin{aligned}
\boldsymbol{q} & =\nabla u & & \text { in } \Omega, \\
-\nabla \cdot \boldsymbol{q} & =f & & \text { in } \Omega, \\
u & =g_{\mathcal{D}} & & \text { on } \Gamma_{\mathcal{D}} \\
\boldsymbol{q} \cdot \boldsymbol{n} & =\boldsymbol{g}_{\mathcal{N}} \cdot \boldsymbol{n} & & \text { on } \Gamma_{\mathcal{N}} .
\end{aligned}
$$

Next, we discretize the above problem on a Cartesian grid $\mathcal{T}$. To obtain the weak formulation with which the LDG is defined, we multiply equations (2.1) and (2.2) by arbitrary, smooth test functions $\boldsymbol{r}$ and $v$, respectively, and integrate by parts over the $d$-dimensional rectangle $K \in \mathcal{T}$. Then we replace the exact solution $(\boldsymbol{q}, u)$ by its approximation $\left(\boldsymbol{q}_{N}, u_{N}\right)$ in the finite element space $\boldsymbol{M}_{N} \times V_{N}$, where

$$
\begin{aligned}
& \boldsymbol{M}_{N}:=\left\{\boldsymbol{q} \in\left(L^{2}(\Omega)\right)^{d}:\left.\boldsymbol{q}\right|_{K} \in \mathcal{S}(K)^{d}, \forall K \in \mathcal{T}\right\}, \\
& V_{N}:=\left\{u \in L^{2}(\Omega):\left.u\right|_{K} \in \mathcal{S}(K), \forall K \in \mathcal{T}\right\}
\end{aligned}
$$

and

$$
\mathcal{S}(K):=\mathcal{Q}^{k}(K)=\{\text { polynomials of degree at most } k \text { in each variable on } K\} .
$$


The method consists in finding $\left(\boldsymbol{q}_{N}, u_{N}\right) \in \boldsymbol{M}_{N} \times V_{N}$ such that

$$
\begin{aligned}
& \int_{K} \boldsymbol{q}_{N} \cdot \boldsymbol{r} d \boldsymbol{x}=-\int_{K} u_{N} \nabla \cdot \boldsymbol{r} d \boldsymbol{x}+\int_{\partial K} \widehat{u}_{N} \boldsymbol{r} \cdot \boldsymbol{n} d s, \\
& \int_{K} \boldsymbol{q}_{N} \cdot \nabla v d \boldsymbol{x}=\int_{K} f v d \boldsymbol{x}+\int_{\partial K} v \widehat{\boldsymbol{q}}_{N} \cdot \boldsymbol{n} d s,
\end{aligned}
$$

for all test functions $(\boldsymbol{r}, v) \in S(K)^{d} \times S(K)$, for all elements $K \in \mathcal{T}$. The functions $\widehat{u}_{N}$ and $\widehat{\boldsymbol{q}}_{N}$ in (2.5) and (2.6) are the so-called numerical fluxes. These are nothing but discrete approximations to the traces of $u$ and $\boldsymbol{q}$ on the boundary of the elements $K$ and are defined as follows. Consider a face $e$ of the $d$-dimensional rectangle $K$. If $e$ lies inside the domain $\Omega$, we define

$$
\left[\begin{array}{l}
\widehat{\boldsymbol{q}}_{N} \\
\widehat{u}_{N}
\end{array}\right]:=\left[\begin{array}{l}
\left\{\boldsymbol{q}_{N}\right\} \\
\left\{u_{N}\right\}
\end{array}\right]-\left[\begin{array}{cc}
C_{11} & \boldsymbol{C}_{12} \\
-\boldsymbol{C}_{12} & 0
\end{array}\right]\left[\begin{array}{l}
\llbracket u_{N} \rrbracket \\
\llbracket \boldsymbol{q}_{N} \rrbracket
\end{array}\right],
$$

and, if $e$ lies on the boundary of $\Omega$,

$$
\widehat{\boldsymbol{q}}:=\left\{\begin{array}{ll}
\boldsymbol{q}^{+}-C_{11}\left(u^{+}-g_{\mathcal{D}}\right) \boldsymbol{n} & \text { on } \Gamma_{\mathcal{D}}, \\
\boldsymbol{g}_{\mathcal{N}} & \text { on } \Gamma_{\mathcal{N}},
\end{array} \quad \text { and } \quad \widehat{u}:= \begin{cases}g_{\mathcal{D}} & \text { on } \Gamma_{\mathcal{D}} \\
u^{+} & \text {on } \Gamma_{\mathcal{N}}\end{cases}\right.
$$

Moreover, the stabilization parameter $C_{11}$ and the auxiliary parameter $\boldsymbol{C}_{12}$ are defined as follows:

$$
C_{11}(e)=\zeta, \quad \boldsymbol{C}_{12}(e) \cdot \boldsymbol{n}=\operatorname{sign}(\boldsymbol{v} \cdot \boldsymbol{n}) / 2,
$$

where $\zeta$ is a positive real number and $\boldsymbol{v}$ is an arbitrary but fixed vector $\boldsymbol{v}$ with non zero components; see Fig. 3.1.

2.2. Error analysis on Cartesian grids. To state our main result, we need to recall some notation and to introduce new hypotheses. We restrict our analysis to domains $\Omega$ such that, for smooth data, the solution $u$ of problem (1.1) belongs to $H^{2}(\Omega)$, and such that when $f$ is in $\mathrm{L}^{2}(\Omega)$ and the boundary data are zero, we have the elliptic regularity result $\|u\|_{2} \leq C\|f\|_{0}$; see Grisvard [8] or [9]. Since the domain $\Omega$ will be triangulated by means of a Cartesian grid, the above requirements hold only if $\Omega$ is a $d$-dimensional rectangle.

We denote by $h_{K}$ the diameter of an element $K$, and set, as usual $h:=\max _{K \in \mathcal{T}} h_{K}$. We denote by $\mathcal{E}_{\mathcal{I}}$ the set of all interior faces of the triangulation $\mathcal{T}$, by $\mathcal{E}_{\mathcal{D}}$ the set of faces on $\Gamma_{\mathcal{D}}$, and by $\mathcal{E}_{\mathcal{N}}$ the set of faces on $\Gamma_{\mathcal{N}}$; we assume that $\bar{\Gamma}_{\mathcal{D}}=\cup_{e \in \mathcal{E}_{\mathcal{D}}} \bar{e}$ and $\bar{\Gamma}_{\mathcal{N}}=\cup_{e \in \mathcal{E}_{\mathcal{N}}} \bar{e}$. The Cartesian triangulations we consider are regular, that is, if $\rho_{K}$ denotes the radius of the biggest ball included in $K$,

$$
\frac{h_{K}}{\rho_{K}} \leq \sigma \quad \forall K \in \mathcal{T} .
$$

Finally, we denote by $E_{\mathcal{N}} \subset \bar{\Omega}$ a closed set containing the intersection between the Neumann boundary $\Gamma_{\mathcal{N}}$ and the set $\{\boldsymbol{x} \in \Gamma: \boldsymbol{v} \cdot \boldsymbol{n}(\boldsymbol{x})<0\}$. Moreover, we assume that the triangulation $\mathcal{T}$ is such that

$$
\bigcup_{e \in \mathcal{E}_{\mathcal{N}}} K_{e} \subset E_{\mathcal{N}}
$$

where $K_{e}$ denotes, from now on, an element containing the face $e$. 
We are now ready to state our main result.

THEOREM 2.1. Assume that the solution $(\boldsymbol{q}, u)$ of $(2.1)-(2.4)$ belongs to $H^{k+1}(\Omega)^{d} \times$ $H^{k+2}(\Omega)$ for $k \geq 0$; assume also that if the intersection between $\Gamma_{\mathcal{N}}$ and $\{\boldsymbol{x} \in \Gamma$ : $\boldsymbol{v} \cdot \boldsymbol{n}(\boldsymbol{x})<0\}$ is non-empty, $u$ belongs to $W^{k+1, \infty}\left(E_{\mathcal{N}}\right)$. Assume that the Cartesian grid $\mathcal{T}$ is shape-regular, (2.12), and that it satisfies the condition (2.13) if $\Gamma_{\mathcal{N}} \cap\{\boldsymbol{x} \in$ $\Gamma: \boldsymbol{v} \cdot \boldsymbol{n}(\boldsymbol{x})<0\} \neq \emptyset$. Let $\left(\boldsymbol{q}_{N}, u_{N}\right) \in \boldsymbol{M}_{N} \times V_{N}$ be the approximation of $(\boldsymbol{q}, u)$ given by the LDG method with $k \geq 0$ and numerical fluxes defined by (2.9), (2.11) and by (2.10).

Then we have

$$
\left\|u-u_{N}\right\|_{0} \leq C h^{k+1}
$$

and

$$
\left|\left(\boldsymbol{q}-\boldsymbol{q}_{N}, u-u_{N}\right)\right|_{\mathcal{A}} \leq C h^{k+\frac{1}{2}},
$$

where the constant $C$ solely depends on $\zeta, k, d, \sigma$ and on the norms $\|u\|_{k+2}$ and $\|u\|_{W^{k+1, \infty}\left(E_{\mathcal{N}}\right)}$.

Several important remarks are in order before we prove this result in the next Section. REMARK 2.2. This theorem is an extension to the bounded domain case of the corresponding result by Cockburn and Shu [7] for the LDG method for transient convection-diffusion problems. It is also an extension to the multi-dimensional case of the results obtained by Castillo, Cockburn, Schötzau and Schwab [4] in the one-space dimension case. The key ingredient of its proof is a super-convergence result of LeSaint and Raviart [10] used in their study of the original DG method for steady-state linear transport in Cartesian grids.

Remark 2.3. Note that Theorem 2.1 holds true in the case $k=0$, that is, when approximate solution is piecewise constant. In [3], all the error estimates obtained for the corresponding LDG method on general grids are valid only for $k \geq 1$; moreover, no order of convergence is numerically observed for $k=0$.

REMARK 2.4. From an approximation point of view, the order of convergence in $\boldsymbol{q}$, namely, $k+1 / 2$, is suboptimal by one half; however, it is confirmed to be sharp by our numerical experiments in Section 4. For general numerical fluxes and unstructured grids, an order of convergence in $\boldsymbol{q}$ of only $k$ is obtained; see [3].

REMARK 2.5. If we take the more general case

$$
C_{11}(e)=\zeta \begin{cases}\min \left\{h_{K_{e}^{+}}, h_{K_{e}^{-}}\right\}^{\alpha}, & \text { if } K_{e}^{+} \cap K_{e}^{-}=e, \\ h_{K_{e}}, & \text { if } e \subset \Gamma,\end{cases}
$$

where $\zeta>0$ and $\alpha$ are constants, we might conceive the possibility that a suitable tuning of the value of $\alpha$ could improve the order of convergence in $\boldsymbol{q}$. However, this is not true, as will be made clear in the proof of Theorem 2.1 displayed in the next section. See also [3] for other results about the influence of the value of $\alpha$ on the orders of convergence of the general LDG method.

REMARK 2.6. In Theorem 2.1 an extra regularity condition on the exact solution $u$ on the closed set $E_{\mathcal{N}}$ containing part of the Neumann boundary is required. If this condition is dropped, and if $\Gamma_{\mathcal{N}} \cap\{\boldsymbol{x} \in \Gamma: \boldsymbol{v} \cdot \boldsymbol{n}(\boldsymbol{x})<0\}$ is not empty, only an order of convergence of $k$ in the error in $\boldsymbol{q}$ can be proved by using our technique which represents a loss of $1 / 2$. Note that whenever it is possible to choose $\boldsymbol{v}$ in such a way that $\Gamma_{\mathcal{N}} \cap\{\boldsymbol{x} \in \Gamma: \boldsymbol{v} \cdot \boldsymbol{n}(\boldsymbol{x})<0\}=\emptyset$, no extra regularity assumption on the exact solution is required. 
3. Proofs. This section is devoted to the proof of Theorem 2.1. For simplicity, we consider only the case $d=2$ and $\Omega$ rectangle; see Fig. 3.1. All the arguments we present in our analysis rely on tensor product structures and can be easily extended to the case $d>2$.

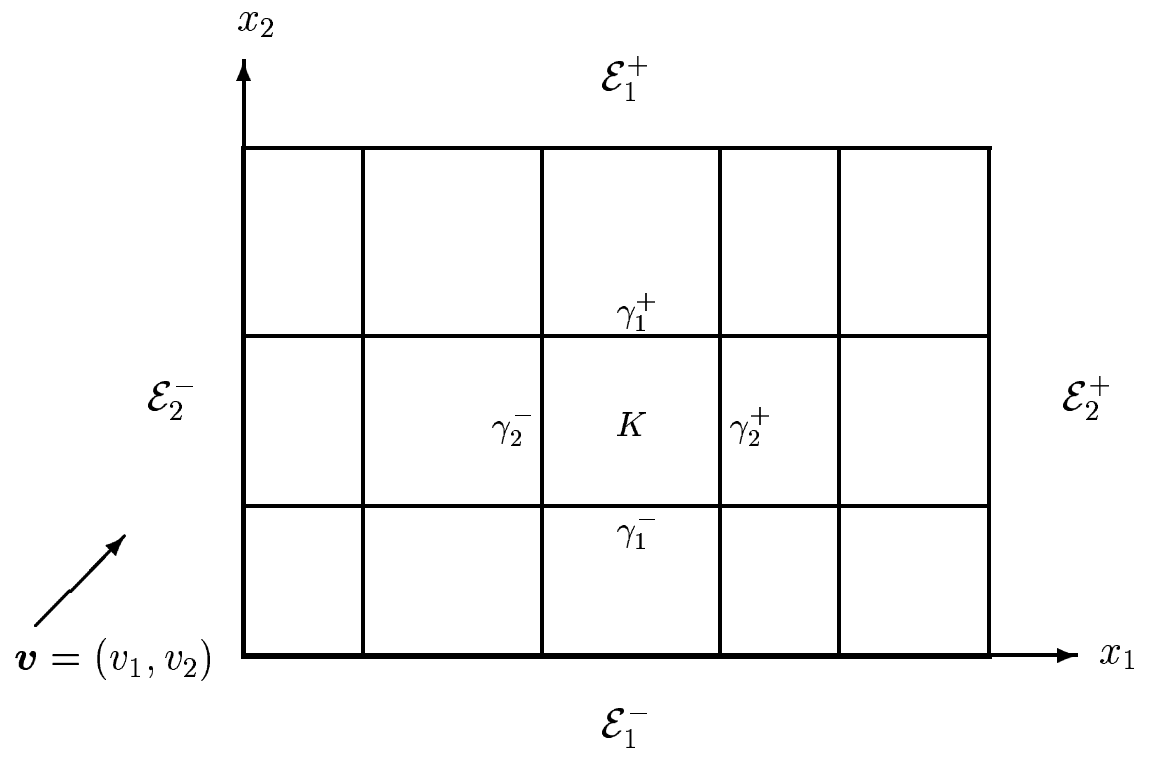

FIG. 3.1. The Cartesian grid $\mathcal{T}$ and the auxiliary vector $\boldsymbol{v}$ used to define the numerical fluxes.

To prove Theorem 2.1, we follow the approach used by [3]. Thus, we start, in Section 3.1 , by briefly reviewing the setting of our error analysis. We proceed in Section 3.2 , by introducing the projections $\Pi$ and $\Pi$ which generalize to several space dimensions the projections used by Castillo, Cockburn, Schötzau and Schwab [4] in their study of the LDG method for transient convection-diffusion problems in onespace dimension. Then, in Section 3.3, we derive the expressions of the functionals $K_{\mathcal{A}}$ and $K_{\mathcal{B}}$ needed in the setting of [3] to get error estimates. To do so, we make use of a super-convergence result essentially due to LeSaint and Raviart [10], and whose proof is presented in Section 3.4. The proof of Theorem 2.1 is completed in Section 3.5.

3.1. The framework of the error analysis. All the following results are collected from [3]. First, we start by reviewing that, by summation over all elements, the LDG method can be written in the compact form: Find $\left(\boldsymbol{q}_{N}, u_{N}\right) \in \boldsymbol{M}_{N} \times V_{N}$ such that

$$
\mathcal{A}\left(\boldsymbol{q}_{N}, u_{N} ; \boldsymbol{r}, v\right)=\mathcal{F}(\boldsymbol{r}, v)
$$

for all $(\boldsymbol{r}, v) \in \boldsymbol{M}_{N} \times V_{N}$, by setting

$$
\begin{aligned}
\mathcal{A}(\boldsymbol{q}, u ; \boldsymbol{r}, v) & :=a(\boldsymbol{q}, \boldsymbol{r})+b(u, \boldsymbol{r})-b(v, \boldsymbol{q})+c(u, v), \\
\mathcal{F}(\boldsymbol{r}, v) & :=F(\boldsymbol{r})+G(v)
\end{aligned}
$$


with

$$
\begin{aligned}
a(\boldsymbol{q}, \boldsymbol{r}) & :=\int_{\Omega} \boldsymbol{q} \cdot \boldsymbol{r} d \boldsymbol{x}, \\
b(u, \boldsymbol{r}) & :=\sum_{K \in \mathcal{T}} \int_{K} u \nabla \cdot \boldsymbol{r} d \boldsymbol{x}-\sum_{e \in \mathcal{E}_{\mathcal{I}}} \int_{e}\left(\{u\}+\boldsymbol{C}_{12} \cdot \llbracket u \rrbracket\right) \llbracket \boldsymbol{r} \rrbracket d s-\sum_{e \in \mathcal{E}_{\mathcal{N}}} \int_{e} u \boldsymbol{r} \cdot \boldsymbol{n} d s, \\
c(u, v) & :=\sum_{e \in \mathcal{E}_{\mathcal{I}}} \int_{e} C_{11} \llbracket u \rrbracket \cdot \llbracket v \rrbracket d s+\sum_{e \in \mathcal{E}_{\mathcal{D}}} \int_{e} C_{11} u v d s .
\end{aligned}
$$

The linear forms $F, G$ are defined by

$$
\begin{aligned}
F(\boldsymbol{r}) & :=\sum_{e \in \mathcal{E}_{\mathcal{D}}} \int_{e} g_{\mathcal{D}} \boldsymbol{r} \cdot \boldsymbol{n} d \boldsymbol{x} \\
G(v) & :=\int_{\Omega} f v d \boldsymbol{x}+\sum_{e \in \mathcal{E}_{\mathcal{D}}} \int_{e} C_{11} g_{\mathcal{D}} v d s+\sum_{e \in \mathcal{E}_{\mathcal{N}}} \int_{e} v \boldsymbol{g}_{\mathcal{N}} \cdot \boldsymbol{n} d s .
\end{aligned}
$$

We also introduce the semi-norm $|(\boldsymbol{q}, u)|_{\mathcal{A}}^{2}$ that appears in a natural way in the analysis of the LDG method and is defined as

$$
|(\boldsymbol{q}, u)|_{\mathcal{A}}^{2}:=\|\boldsymbol{q}\|_{0}^{2}+\sum_{e \in \mathcal{E}_{\mathcal{I}}} \int_{e} C_{11} \llbracket u \rrbracket^{2} d s+\sum_{e \in \mathcal{E}_{\mathcal{D}}} \int_{e} C_{11} u^{2} d s .
$$

To prove error estimates for the LDG method, we follow [3] and introduce two functionals, $K_{\mathcal{A}}$ and $K_{\mathcal{B}}$, which capture the approximation properties of the LDG method; the functionals are related to two suitably chosen projections $\Pi$ and $\Pi$ onto the $\mathrm{FE}$ spaces $\boldsymbol{M}_{N}$ and $V_{N}$, respectively. Namely, we require $K_{\mathcal{A}}$ and $K_{\mathcal{B}}$ to satisfy

$$
|\mathcal{A}(\boldsymbol{q}-\Pi \boldsymbol{q}, u-\Pi u ; \boldsymbol{\Phi}-\Pi \boldsymbol{\Phi}, \varphi-\Pi \varphi)| \leq K_{\mathcal{A}}(\boldsymbol{q}, u ; \boldsymbol{\Phi}, \varphi)
$$

for any $(\boldsymbol{q}, u),(\boldsymbol{\Phi}, \varphi) \in H^{1}(\Omega)^{d} \times H^{2}(\Omega)$, and

$$
|\mathcal{A}(\boldsymbol{r}, v ; \boldsymbol{q}-\Pi \boldsymbol{q}, u-\Pi u)| \leq|(\boldsymbol{r}, v)|_{\mathcal{A}} K_{\mathcal{B}}(\boldsymbol{q}, u)
$$

for any $(\boldsymbol{r}, v) \in \boldsymbol{M}_{N} \times V_{N}$ and $(\boldsymbol{q}, u) \in H^{1}(\Omega)^{d} \times H^{2}(\Omega)$.

By Galerkin orthogonality, all the error estimates can then be solely expressed in terms of $K_{\mathcal{A}}$ and $K_{\mathcal{B}}$ as can be seen in the following result.

LEMMA 3.1 ([3]). We have

$$
\left|\left(\boldsymbol{q}-\boldsymbol{q}_{N}, u-u_{N}\right)\right|_{\mathcal{A}} \leq K_{\mathcal{A}}^{1 / 2}(\boldsymbol{q}, u ; \boldsymbol{q}, u)+K_{\mathcal{B}}(\boldsymbol{q}, u) .
$$

Furthermore,

$$
\left\|u-u_{N}\right\|_{0} \leq \sup _{\lambda \in L^{2}(\Omega)} \frac{K_{\mathcal{A}}(\boldsymbol{q}, u ; \boldsymbol{\Phi}, \varphi)}{\|\lambda\|_{0}}+K_{\mathcal{B}}(\boldsymbol{q}, u) \sup _{\lambda \in L^{2}(\Omega)} \frac{K_{\mathcal{B}}(\boldsymbol{\Phi}, \varphi)}{\|\lambda\|_{0}},
$$

with $\varphi$ denoting the solution of the adjoint problem

$$
\begin{aligned}
-\Delta \varphi & =\lambda & & \text { in } \Omega, \\
\varphi & =0 & & \text { on } \Gamma_{\mathcal{D}}, \\
\frac{\partial \varphi}{\partial n} & =0 & & \text { on } \Gamma_{\mathcal{N}}
\end{aligned}
$$

and $\boldsymbol{\Phi}=-\nabla \varphi$. 
3.2. Projections. In this section we define the projections $\Pi$ and $\Pi$ we are going to use to prove Theorem 2.1 and list their properties. To this end, we start by introducing one-dimensional projections. Let $I=\left(a^{-}, a^{+}\right)$be an arbitrary interval, and let $\mathcal{P}^{k}(I)$ be the space of the polynomials of degree at most $k$ on $I$. We denote by $\pi$ the $\mathrm{L}^{2}(I)$-projection onto $\mathcal{P}^{k}(I)$, i.e., for a function $w \in \mathrm{L}^{2}(I)$ the projection $\pi w$ is the unique polynomial in $\mathcal{P}^{k}(I)$ satisfying

$$
\int_{I}[w(x)-\pi w(x)] p(x) d x=0 \quad \forall p \in \mathcal{P}^{k}(I) .
$$

Furthermore, for $w \in H^{\frac{1}{2}+\varepsilon}(I)$, we define the projections $\pi^{ \pm} w \in \mathcal{P}^{k}(I)$ by the following $k+1$ conditions:

$$
\begin{aligned}
& \int_{I}\left[w(x)-\pi^{ \pm} w(x)\right] p(x) d x=0 \quad \forall p \in \mathcal{P}^{k-1}(I), \\
& \pi^{ \pm} w\left(a^{ \pm}\right)=w\left(a^{ \pm}\right) .
\end{aligned}
$$

On a rectangle $K=I_{1} \times I_{2}$, we define the following tensor product operators:

$$
\begin{aligned}
\Pi^{ \pm} v & :=\pi_{1}^{ \pm} \otimes \pi_{2}^{ \pm} v, \\
\Pi^{ \pm} r & :=\left(\pi_{1}^{ \pm} \otimes \pi_{2} r_{1}, \pi_{1} \otimes \pi_{2}^{ \pm} r_{2}\right),
\end{aligned}
$$

with the subscripts indicating the application of the one-dimensional operators $\pi$ or $\pi^{ \pm}$with respect to the corresponding variable.

Finally, we define the projections $\Pi$ and $\Pi$ as

$$
\Pi \boldsymbol{q}_{\mid K}:=\Pi^{-} \boldsymbol{q}_{\mid K}, \quad \Pi u_{\mid K}:=\Pi^{+} u_{\mid K}, \quad \forall K \in \mathcal{T} .
$$

In our error analysis, we use key properties of these projections displayed in the following result.

Lemma 3.2. With the notation indicated in Figure 3.1, we have

$$
\begin{array}{ll}
\int_{K}\left[\boldsymbol{r}-\boldsymbol{\Pi}^{ \pm} \boldsymbol{r}\right] \cdot \nabla p d \boldsymbol{x}=0 & \forall p \in \mathcal{Q}^{k}(K), \\
\int_{\gamma_{i}^{ \pm}}\left[\boldsymbol{r}-\boldsymbol{\Pi}^{ \pm} \boldsymbol{r}\right] \cdot \boldsymbol{n} p d s=0 & \forall p \in \mathcal{P}^{k}\left(\gamma_{i}^{ \pm}\right), i=1,2 .
\end{array}
$$

We also need several approximation results which we gather in the lemma below. Lemma 3.3 (Cf. [5]). Let $v \in H^{s+2}(K)$ and $r \in H^{s+1}(K)^{2}, s \geq 0$. Then for $m$ integer, $0 \leq m \leq s+1$, we have

$$
\begin{aligned}
& \left|v-\Pi^{ \pm} v\right|_{m, K} \leq C h_{K}^{\min \{s+1, k\}+1-m}\|v\|_{s+2, K}, \\
& \left\|v-\Pi^{ \pm} v\right\|_{0, e} \leq C h_{K}^{\min \{s+1, k\}+\frac{1}{2}}\|v\|_{s+2, K}, \quad \forall e \subset \partial K, \\
& \left|\boldsymbol{r}-\boldsymbol{\Pi}^{ \pm} \boldsymbol{r}\right|_{m, K} \leq C h_{K}^{\min \{s, k\}+1-m}\|\boldsymbol{r}\|_{s+1, K}, \\
& \left\|\boldsymbol{r}-\boldsymbol{\Pi}^{ \pm} \boldsymbol{r}\right\|_{0, e} \leq C h_{K}^{\min \{s, k\}+\frac{1}{2}}\|\boldsymbol{r}\|_{s+1, K}, \quad \forall e \subset \partial K .
\end{aligned}
$$

Furthermore, for any edge $e_{i}$ parallel to the $x_{i}$-axis, $i=1,2$, we have

$$
\left\|w-\pi_{i}^{ \pm} w\right\|_{0, e_{i}} \leq C h_{e_{i}}^{\min \left\{s+\frac{1}{2}, k\right\}+1}\|w\|_{s+\frac{3}{2}, e_{i}}, \quad \forall w \in H^{s+\frac{3}{2}}\left(e_{i}\right) .
$$

Finally, if $u \in W^{s+1, \infty}(K)$, then

$$
\left\|v-\Pi^{ \pm} v\right\|_{L^{\infty}(e)} \leq C h_{K}^{\min \{s, k\}+1}\|v\|_{W^{s+1, \infty}(K)}, \quad \forall e \subset \partial K .
$$


3.3. The functionals $K_{\mathcal{A}}$ and $K_{\mathcal{B}}$. In this subsection, we obtain the functionals $K_{\mathcal{A}}$ and $K_{\mathcal{B}}$ introduced in Section 3.1.

We consider the stabilization parameter $C_{11}$, defined by (2.14), in order to highlight the fact that any choice of $\alpha \neq 0$ in (2.14) deteriorates the rates of convergence of the estimates of Theorem 2.1.

In [3, Corollary 3.4], $K_{\mathcal{A}}$ has been investigated for general projection operators $\Pi$ and $\Pi$ satisfying the approximation results in Lemma 3.3 with $m=0,1$. Thus, we we just report here the final result.

Lemma 3.4 ([3]). Let $u \in H^{s+2}(\Omega), s \geq 0$, and $\varphi \in H^{t+2}(\Omega), t \geq 0$. Assume $C_{11}$ to be given by (2.14). Then, if we set $\boldsymbol{q}=\nabla u$ and $\boldsymbol{\Phi}= \pm \nabla \varphi$, the approximation property (3.2) holds true with

$$
\begin{aligned}
K_{\mathcal{A}}(\boldsymbol{q}, u ; \boldsymbol{\Phi}, \varphi)= & {\left[h^{\min \{s, k\}+1}\left(h^{\min \{t, k\}+1}+h^{\min \{t+1, k\}}\right)\right.} \\
& \left.+h^{\min \{s+1, k\}+1}\left(h^{\min \{t, k\}}+\zeta h^{\min \{t+1, k\}+\alpha}\right)\right]\|u\|_{s+2}\|\varphi\|_{t+2} .
\end{aligned}
$$

Furthermore, in the particular case where $(\boldsymbol{\Phi}, \varphi)=(\boldsymbol{q}, u)$, there holds

$$
K_{\mathcal{A}}(\boldsymbol{q}, u ; \boldsymbol{q}, u)=\left[h^{2 \min \{s, k\}+2}+\zeta h^{2 \min \{s+1, k\}+1+\alpha}\right]\|u\|_{s+2}^{2} .
$$

In [3], the functional $K_{\mathcal{B}}$ was only studied in the case where $\Pi$ and $\Pi$ are $\mathrm{L}^{2}$ projections. Next, we show that a better result for $K_{\mathcal{B}}$ can be obtained on Cartesian grids for the projections defined by (3.4) and the numerical fluxes defined by (2.11). To obtain such a result, we use the following standard inverse inequality.

Lemma 3.5 (Cf. [5]). There exists a positive constant $C$ solely depending on $k, d$ and $\sigma$ such that for all $s \in \boldsymbol{M}_{N}$ we have

$$
\|s\|_{0, e} \leq C h_{K}^{-\frac{1}{2}}\|s\|_{0, K}
$$

for all $K \in \mathcal{T}$, e being any side of $K$.

We set $u_{\Gamma}=\left.u\right|_{\Gamma}$, and $\left\|u_{\Gamma}\right\|_{s+\frac{3}{2}, \Gamma}^{2}=\sum_{j=1}^{4}\left\|u_{\Gamma}\right\|_{s+\frac{3}{2}, \Gamma_{j}}^{2}$, where $\Gamma_{j}, j=1,2,3,4$, denote the sides of $\Gamma$. We are now ready to state our main lemma.

Lemma 3.6. Let $u \in H^{s+2}(\Omega), s \geq 0$, and set $\boldsymbol{q}=\nabla u$. Assume $C_{11}$ to be given by (2.14) and let $\Pi$ and $\Pi$ be the operators defined by (3.4). Then, for any $(\boldsymbol{r}, w) \in$ $\boldsymbol{M}_{N} \times V_{N}$, the approximation property (3.3) holds true, with $K_{\mathcal{B}}$ given by

$$
\begin{aligned}
K_{\mathcal{B}}(\boldsymbol{q}, u):= & C\left[h^{\min \{s, k\}+1}+\zeta^{-\frac{1}{2}} h^{\min \{s, k\}+\frac{1}{2}-\frac{\alpha}{2}}+\zeta^{\frac{1}{2}} h^{\min \{s+1, k\}+\frac{1}{2}+\frac{\alpha}{2}}\right]\|u\|_{s+2} \\
& +C\left|\mathcal{E}_{\mathcal{N}} \cap \mathcal{E}^{-}\right|^{\frac{1}{2}} h^{\min \{s, k\}+\frac{1}{2}}\|u\|_{W^{s+1, \infty}\left(E_{\mathcal{N}}\right)}+C h^{\min \left\{s+\frac{1}{2}, k\right\}+\frac{1}{2}}\left\|u_{\Gamma}\right\|_{s+\frac{3}{2}, \Gamma},
\end{aligned}
$$

where the constant $C$ solely depends on $k, d$ and $\sigma$.

Proof. In order to be able to distinguish the many parts of $\Gamma$ and facilitate the proof of the above result, we introduce the following notation:

$$
\begin{array}{ll}
\mathcal{E}^{+}:=\{e \subset \Gamma: \boldsymbol{v} \cdot \boldsymbol{n}>0\}, & \mathcal{E}^{-}:=\{e \subset \Gamma: \boldsymbol{v} \cdot \boldsymbol{n}<0\}, \\
\mathcal{E}_{1}^{+}:=\left\{e \in \mathcal{E}^{+} \mid x_{2}=\mathrm{const}\right\}, & \mathcal{E}_{1}^{-}:=\left\{e \in \mathcal{E}^{-} \mid x_{2}=\mathrm{const}\right\}, \\
\mathcal{E}_{2}^{+}:=\left\{e \in \mathcal{E}^{+} \mid x_{1}=\mathrm{const}\right\}, & \mathcal{E}_{2}^{-}:=\left\{e \in \mathcal{E}^{-} \mid x_{1}=\mathrm{const}\right\},
\end{array}
$$

and define $\mathcal{E}_{i}:=\mathcal{E}_{i}^{+} \cup \mathcal{E}_{i}^{-}$; these boundaries are indicated in Fig. 3.1.

We set $\boldsymbol{\xi}_{\boldsymbol{q}}:=\boldsymbol{q}-\Pi \boldsymbol{q}$ and $\xi_{u}:=u-\Pi u$, write

$$
\left|\mathcal{A}\left(\boldsymbol{r}, v ; \boldsymbol{\xi}_{\boldsymbol{q}}, \xi_{u}\right)\right| \leq\left|a\left(\boldsymbol{r}, \boldsymbol{\xi}_{\boldsymbol{q}}\right)\right|+\left|b\left(v, \boldsymbol{\xi}_{\boldsymbol{q}}\right)\right|+\left|b\left(\xi_{u}, \boldsymbol{r}\right)\right|+\left|c\left(\xi_{u}, v\right)\right|=: T_{1}+T_{2}+T_{3}+T_{4}
$$


and estimate each of the forms separately.

a. Estimate of $T_{1}$. We have

$$
\begin{aligned}
T_{1} & =\left|\sum_{K \in \mathcal{T}} \int_{K} \boldsymbol{r} \cdot \boldsymbol{\xi}_{\boldsymbol{q}} d \boldsymbol{x}\right| \leq\left(\sum_{K \in \mathcal{T}}\|\boldsymbol{r}\|_{0, K}^{2}\right)^{\frac{1}{2}}\left(\sum_{K \in \mathcal{T}}\left\|\boldsymbol{\xi}_{\boldsymbol{q}}\right\|_{0, K}^{2}\right)^{\frac{1}{2}} \\
& \leq C|(\boldsymbol{r}, v)|_{\mathcal{A}}\left(\sum_{K \in \mathcal{T}} h_{K}^{2 \min \{s, k\}+2}\|\boldsymbol{q}\|_{s+1, K}^{2}\right)^{\frac{1}{2}} .
\end{aligned}
$$

b. Estimate of $T_{2}$. We can write

$T_{2}=\mid-\sum_{K \in \mathcal{T}} \int_{K} \nabla v \cdot \boldsymbol{\xi}_{\boldsymbol{q}} d \boldsymbol{x}+\sum_{e \in \mathcal{E}_{\mathcal{I}}} \int_{e} \llbracket v \rrbracket \cdot\left(\left\{\left\{\boldsymbol{\xi}_{\boldsymbol{q}}\right\}-\boldsymbol{C}_{12} \llbracket \boldsymbol{\xi}_{\boldsymbol{q}} \rrbracket\right) d s+\sum_{e \in \mathcal{E}_{\mathcal{D}}} \int_{e} v \boldsymbol{\xi}_{\boldsymbol{q}} \cdot \boldsymbol{n} d s \mid\right.$.

Taking into account the definition of the fluxes in (2.11) and the properties of the projection $\boldsymbol{\Pi}$ in Lemma 3.2, we conclude that

$$
\begin{aligned}
& \int_{K} \nabla v \cdot \boldsymbol{\xi}_{\boldsymbol{q}} d \boldsymbol{x}=0, \forall K \in \mathcal{T}, \quad \int_{e} v \boldsymbol{\xi}_{\boldsymbol{q}} \cdot \boldsymbol{n} d s=0, \forall e \in \mathcal{E}^{-}, \\
& \int_{e} \llbracket v \rrbracket \cdot\left(\left\{\left\{\boldsymbol{\xi}_{\boldsymbol{q}}\right\}-\boldsymbol{C}_{12} \llbracket \boldsymbol{\xi}_{\boldsymbol{q}} \rrbracket\right) d s=0, \forall e \in \mathcal{E}_{\mathcal{I}} .\right.
\end{aligned}
$$

Consequently,

$$
T_{2}=\left|\sum_{e \in \mathcal{E}^{+} \cap \mathcal{E}_{\mathcal{D}}} \int_{e} v \boldsymbol{\xi}_{\boldsymbol{q}} \cdot \boldsymbol{n} d s\right|
$$

Multiplying and dividing each term of the sum by $C_{11}^{\frac{1}{2}}$, and using the approximation properties of $\boldsymbol{\Pi}$, we have

$$
\begin{aligned}
T_{2} & =\left|\sum_{e \in \mathcal{E}^{+} \cap \mathcal{E}_{\mathcal{D}}} \int_{e} v \boldsymbol{\xi}_{\boldsymbol{q}} \cdot \boldsymbol{n} d s\right| \leq\left(\sum_{e \in \mathcal{E}^{+} \cap \mathcal{E}_{\mathcal{D}}} C_{11}\|v\|_{0, e}^{2}\right)^{\frac{1}{2}}\left(\sum_{e \in \mathcal{E}^{+} \cap \mathcal{E}_{\mathcal{D}}} C_{11}^{-1}\left\|\boldsymbol{\xi}_{\boldsymbol{q}}\right\|_{0, e}^{2}\right)^{\frac{1}{2}} \\
& \leq C|(\boldsymbol{r}, v)|_{\mathcal{A}}\left(\zeta^{-1} \sum_{e \in \mathcal{E}^{+} \cap \mathcal{E}_{\mathcal{D}}} h_{K_{e}}^{2 \min \{s, k\}+1-\alpha}\|\boldsymbol{q}\|_{s+1, K_{e}}^{2}\right)^{\frac{1}{2}} .
\end{aligned}
$$

Note that we have used the shape-regularity assumption (2.12) to bound $C_{11}^{-1}$ by $C \zeta^{-1} h_{K_{e}}^{-\alpha}$.

c. The estimate of $T_{4}$. We have

$$
\begin{aligned}
T_{4} & =\left|\sum_{e \in \mathcal{E}_{\mathcal{I}}} \int_{e} C_{11} \llbracket v \rrbracket \cdot \llbracket \xi_{u} \rrbracket d s+\sum_{e \in \mathcal{E}_{\mathcal{D}}} \int_{e} C_{11} v \xi_{u} d s\right| \\
& \leq\left(\sum_{e \in \mathcal{E}_{\mathcal{I}}} C_{11}\|\llbracket v \rrbracket\|_{0, e}^{2}+\sum_{e \in \mathcal{E}_{\mathcal{D}}} C_{11}\|v\|_{0, e}^{2}\right)^{\frac{1}{2}} \cdot\left(\sum_{e \in \mathcal{E}_{\mathcal{I}}} C_{11}\left\|\llbracket \xi_{u} \rrbracket\right\|_{0, e}^{2}+\sum_{e \in \mathcal{E}_{\mathcal{D}}} C_{11}\left\|\xi_{u}\right\|_{0, e}^{2}\right)^{\frac{1}{2}} \\
& \leq|(\boldsymbol{r}, v)|_{\mathcal{A}}\left(\sum_{K \in \mathcal{T}} \sum_{e \subset \partial K} C_{11}\left\|\xi_{u}\right\|_{0, e}^{2}\right)^{\frac{1}{2}} \\
& \leq C|(\boldsymbol{r}, v)|_{\mathcal{A}}\left(\zeta \sum_{K \in \mathcal{T}} h_{K}^{2 \min \{s+1, k\}+1+\alpha}\|u\|_{s+2, K}^{2}\right)^{\frac{1}{2}} \\
& \leq C|(\boldsymbol{r}, v)|_{\mathcal{A}}\left(\zeta h^{2 \min \{s+1, k\}+1+\alpha}\|u\|_{s+2}^{2}\right)^{\frac{1}{2}} .
\end{aligned}
$$


d. Estimate of $T_{3}$. This estimate cannot be obtained as easily as the previous ones since it is here that the key idea introduced by LeSaint and Raviart [10] has to be suitably applied.

We start by writing

$$
\begin{aligned}
T_{3}= & \left|\sum_{K \in \mathcal{T}} \int_{K} \xi_{u} \nabla \cdot \boldsymbol{r} d \boldsymbol{x}-\sum_{e \in \mathcal{E}_{\mathcal{I}}} \int_{e}\left(\left\{\xi_{u}\right\}+\boldsymbol{C}_{12} \cdot \llbracket \xi_{u} \rrbracket\right) \llbracket \boldsymbol{r} \rrbracket d s-\sum_{e \in \mathcal{E}_{\mathcal{N}}} \int_{e} \xi_{u} \boldsymbol{r} \cdot \boldsymbol{n} d s\right| \\
= & \mid \sum_{K \in \mathcal{T}} \int_{K} \xi_{u} \nabla \cdot \boldsymbol{r} d \boldsymbol{x}-\sum_{K \in \mathcal{T}}\left(\sum_{\substack{e \subset \partial K \\
e \in \mathcal{E}_{\mathcal{I}}}} \int_{e}\left(\left\{\xi_{u}\right\}+\boldsymbol{C}_{12} \cdot \llbracket \xi_{u} \rrbracket\right) \boldsymbol{r} \cdot \boldsymbol{n} d s\right) \\
& -\sum_{e \in \mathcal{E}_{\mathcal{N}}} \int_{e} \xi_{u} \boldsymbol{r} \cdot \boldsymbol{n} d s \mid .
\end{aligned}
$$

Again with (2.11), we see that the contribution of an interior element $K$ to this expression is

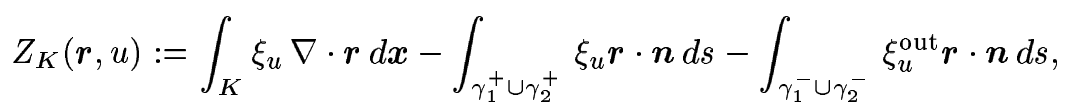

where the superscript 'out' denotes the traces taken from outside $K$. Since $u_{\left.\right|_{\gamma_{i}^{ \pm}} ^{\text {out }}}^{\text {ut }}=u_{\left.\right|_{\gamma_{i}^{ \pm}}}$ and $[\Pi u]_{\gamma_{\gamma_{i}^{-}}}^{\text {out }}=\pi_{i}^{+}\left(u_{\left.\right|_{\gamma_{i}^{-}}}\right)$for the corresponding one-dimensional projection $\pi_{i}^{+}$, this contribution can be written as

$$
\begin{aligned}
Z_{K}(\boldsymbol{r}, u)= & \int_{K}\left(u-\Pi^{+} u\right) \nabla \cdot \boldsymbol{r} d \boldsymbol{x}-\int_{\gamma_{1}^{+}}\left(u-\pi_{1}^{+} u\right) \boldsymbol{r} \cdot \boldsymbol{n} d s-\int_{\gamma_{2}^{+}}\left(u-\pi_{2}^{+} u\right) \boldsymbol{r} \cdot \boldsymbol{n} d s \\
& -\int_{\gamma_{1}^{-}}\left(u-\pi_{1}^{+} u\right) \boldsymbol{r} \cdot \boldsymbol{n} d s-\int_{\gamma_{2}^{-}}\left(u-\pi_{2}^{+} u\right) \boldsymbol{r} \cdot \boldsymbol{n} d s .
\end{aligned}
$$

For boundary elements, we add and subtract corresponding terms to obtain

$$
\begin{aligned}
T_{3} \leq & \left|\sum_{K \in \mathcal{T}} Z_{K}(\boldsymbol{r}, u)\right|+\mid \sum_{e \in \mathcal{E}_{1}} \int_{e}\left(u_{\Gamma}-\pi_{1}^{+} u_{\Gamma}\right) \boldsymbol{r} \cdot \boldsymbol{n} d s+\sum_{e \in \mathcal{E}_{2}} \int_{e}\left(u_{\Gamma}-\pi_{2}^{+} u_{\Gamma}\right) \boldsymbol{r} \cdot \boldsymbol{n} d s \\
& +\sum_{e \in \mathcal{E}_{\mathcal{N}}} \xi_{u} \boldsymbol{r} \cdot \boldsymbol{n} d s \mid \\
\leq & \sum_{K \in \mathcal{T}}\left|Z_{K}(\boldsymbol{r}, u)\right|+\sum_{e \in \mathcal{E}_{1} \backslash\left(\mathcal{E}_{\left.\mathcal{N} \cap \mathcal{E}^{-}\right)} \int_{e}\left|\left(u_{\Gamma}-\pi_{1}^{+} u_{\Gamma}\right) \boldsymbol{r} \cdot \boldsymbol{n}\right| d s+\right.} \\
& +\sum_{e \in \mathcal{E}_{2} \backslash\left(\mathcal{E}_{\mathcal{N}} \cap \mathcal{E}^{-}\right)} \int_{e}\left|\left(u_{\Gamma}-\pi_{1}^{+} u_{\Gamma}\right) \boldsymbol{r} \cdot \boldsymbol{n}\right| d s+\sum_{e \in \mathcal{E}_{\mathcal{N} \cap \mathcal{E}^{-}}} \int_{e}\left|\xi_{u} \boldsymbol{r} \cdot \boldsymbol{n}\right| d s
\end{aligned}
$$

with $Z_{K}(\boldsymbol{r}, u)$ defined in (3.5).

We start by bounding the contributions to $T_{3}$ stemming from a boundary edge $e_{i} \in \mathcal{E}_{i} \backslash$ $\left(\mathcal{E}_{\mathcal{N}} \cap \mathcal{E}^{-}\right)$parallel to the $x_{i}$-axis, $i=1,2$. Since $u \in H^{s+2}(\Omega)$ implies $u_{\Gamma} \in H^{s+\frac{3}{2}}\left(e_{i}\right)$, see [9], by the property (3.3) and the inverse inequality in Lemma 3.5, we get

$$
\begin{aligned}
\int_{e_{i}}\left|\left(u_{\Gamma}-\pi_{i}^{ \pm} u_{\Gamma}\right) \boldsymbol{r} \cdot \boldsymbol{n}\right| d s & \leq\left\|u_{\Gamma}-\pi_{i}^{ \pm} u_{\Gamma}\right\|_{0, e_{i}}\|\boldsymbol{r}\|_{0, e_{i}} \\
& \leq C h_{e_{i}}^{\min \left\{s+\frac{1}{2}, k\right\}+\frac{1}{2}}\left\|u_{\Gamma}\right\|_{s+\frac{3}{2}, e_{i}}\|\boldsymbol{r}\|_{0, K_{e_{i}}} .
\end{aligned}
$$


Here, $K_{e_{i}}$ denotes again the element containing the edge $e_{i}$. Consequently, the global contribution to $T_{3}$ of the boundary edges belonging to $\mathcal{E}_{i} \backslash\left(\mathcal{E}_{\mathcal{N}} \cap \mathcal{E}^{-}\right)$can be bounded by

$$
\begin{gathered}
\sum_{e \in\left(\mathcal{E}_{1} \cup \mathcal{E}_{2}\right) \backslash\left(\mathcal{E}_{\left.\mathcal{N} \cap \mathcal{E}^{-}\right)}\right.} C h_{e}^{\min \left\{s+\frac{1}{2}, k\right\}+\frac{1}{2}}\left\|u_{\Gamma}\right\|_{s+\frac{3}{2}, e}\|\boldsymbol{r}\|_{0, K_{e}} \\
\leq C\left(\sum_{e \subset \Gamma} h_{e}^{2 \min \left\{s+\frac{1}{2}, k\right\}+1}\left\|u_{\Gamma}\right\|_{s+\frac{3}{2}, e}^{2}\right)^{\frac{1}{2}}\left(\sum_{e \subset \Gamma}\|\boldsymbol{r}\|_{0, K_{e}}^{2}\right)^{\frac{1}{2}} \\
\leq C h^{\min \left\{s+\frac{1}{2}, k\right\}+\frac{1}{2}}\left(\sum_{e \subset \Gamma}\left\|u_{\Gamma}\right\|_{s+\frac{3}{2}, e}^{2}\right)^{\frac{1}{2}}|(\boldsymbol{r}, v)|_{\mathcal{A}} \\
\leq C h^{\min \left\{s+\frac{1}{2}, k\right\}+\frac{1}{2}}\left\|u_{\Gamma}\right\|_{s+\frac{3}{2}, \Gamma}|(\boldsymbol{r}, v)|_{\mathcal{A}} .
\end{gathered}
$$

For the edges $e$ in $\mathcal{E}_{\mathcal{N}} \cap \mathcal{E}^{-}$, we have to use a different argument. Thus, by Lemma 3.5, we have

$$
\begin{aligned}
\int_{e}\left|\xi_{u} \boldsymbol{r} \cdot \boldsymbol{n}\right| d s & \leq\left\|\xi_{u}\right\|_{L^{\infty}(e)}\|\boldsymbol{r} \cdot \boldsymbol{n}\|_{L^{1}(e)} \\
& \leq C|e|^{\frac{1}{2}} h_{K_{e}}^{-\frac{1}{2}}\left\|\xi_{u}\right\|_{L^{\infty}(e)}\|\boldsymbol{r}\|_{0, K_{e}}
\end{aligned}
$$

Hence, by the Cauchy-Schwarz inequality,

$$
\sum_{e \in \mathcal{E}_{\mathcal{N}} \cap \mathcal{E}^{-}}\left|\xi_{u} \boldsymbol{r} \cdot \boldsymbol{n}\right| d s \leq C\left|\mathcal{E}_{\mathcal{N}} \cap \mathcal{E}^{-}\right|^{\frac{1}{2}}\left(\sup _{e \in \mathcal{E}_{\mathcal{N}} \cap \mathcal{E}^{-}} h_{K_{e}}^{-\frac{1}{2}}\left\|\xi_{u}\right\|_{L^{\infty}(e)}\right)\|\boldsymbol{r}\|_{0},
$$

and so

$$
\sum_{e \in \mathcal{E}_{\mathcal{N}} \cap \mathcal{E}^{-}}\left|\xi_{u} \boldsymbol{r} \cdot \boldsymbol{n}\right| d s \leq C\left|\mathcal{E}_{\mathcal{N}} \cap \mathcal{E}^{-}\right|^{\frac{1}{2}} h^{\min \{s, k\}+\frac{1}{2}}\|u\|_{W^{s+1, \infty}\left(E_{\mathcal{N}}\right)}|(\boldsymbol{r}, v)|_{\mathcal{A}} .
$$

Finally, we estimate the contribution $Z_{K}(\boldsymbol{r}, u)$, by using the following super-convergence result, essentially due to LeSaint and Raviart [10], whose proof is postponed to Section 3.4 .

Lemma 3.7. Let $Z_{K}(\boldsymbol{r}, u)$ be defined by (3.5). Then we have for $s \geq 0$

$$
\left|Z_{K}(\boldsymbol{r}, u)\right| \leq C h_{K}^{\min \{s, k\}+1}\|u\|_{s+2, K}\|\boldsymbol{r}\|_{0, K} .
$$

By combining the result of Lemma 3.7 with the above estimates of the contribution of boundary edges, we obtain

$$
\begin{aligned}
T_{3} \leq C|(\boldsymbol{r}, v)|_{\mathcal{A}} & \left(h^{\min \{s, k\}+1}\|u\|_{s+2}+h^{\min \left\{s+\frac{1}{2}, k\right\}+\frac{1}{2}}\left\|u_{\Gamma}\right\|_{s+\frac{3}{2}, \Gamma}\right. \\
& \left.+\left|\mathcal{E}_{\mathcal{N}} \cap \mathcal{E}^{-}\right|^{\frac{1}{2}} h^{\min \{s, k\}+\frac{1}{2}}\|u\|_{W^{s+1, \infty}\left(E_{\mathcal{N}}\right)}\right) .
\end{aligned}
$$

Conclusion. The result now follows by simply gathering the estimates for $T_{i}, i=$ $1,2,3,4$, obtained above. This completes the proof. 
3.4. Proof of Lemma 3.7. We can write

$$
Z_{K}(\boldsymbol{r}, u)=Z_{K, 1}\left(r_{1}, u\right)+Z_{K, 2}\left(r_{2}, u\right),
$$

where

$$
\begin{aligned}
Z_{K, 1}\left(r_{1}, u\right)= & \int_{K}\left(u-\Pi^{+} u\right) \frac{\partial r_{1}}{\partial x_{1}} d x_{1} d x_{2}-\int_{\gamma_{2}^{+}}\left(u-\pi_{2}^{+} u\right) r_{1} d x_{2} \\
& +\int_{\gamma_{2}^{-}}\left(u-\pi_{2}^{+} u\right) r_{1} d x_{2}
\end{aligned}
$$

and

$$
\begin{aligned}
Z_{K, 2}\left(r_{2}, u\right)= & \int_{K}\left(u-\Pi^{+} u\right) \frac{\partial r_{2}}{\partial x_{2}} d x_{1} d x_{2}-\int_{\gamma_{1}^{+}}\left(u-\pi_{1}^{+} u\right) r_{2} d x_{1} \\
& +\int_{\gamma_{1}^{-}}\left(u-\pi_{1}^{+} u\right) r_{2} d x_{1} .
\end{aligned}
$$

The proof of the approximation results for $Z_{K, 1}$ and $Z_{K, 2}$ are analogous; therefore, we just present the one for $Z_{K, 1}$, essentially following the same arguments as in [10]. First, we consider $Z_{K, 1}$ on the reference square $(-1,1)^{2}$. We claim that

$$
Z_{K, 1}\left(r_{1}, u\right)=0 \quad \forall u \in \mathcal{P}^{k+1}(K), r_{1} \in \mathcal{Q}^{k}(K) .
$$

To prove (3.6), fix $r_{1} \in \mathcal{Q}^{k}(K)$. Since $\Pi^{+}$and $\pi^{+}$are polynomial preserving operators, (3.6) holds true for every $u \in \mathcal{Q}^{k}(K)$. Therefore, we just have to consider the cases $u\left(x_{1}, x_{2}\right)=x_{1}^{k+1}$ and $u\left(x_{1}, x_{2}\right)=x_{2}^{k+1}$.

Let us start with $u\left(x_{1}, x_{2}\right)=x_{1}^{k+1}$. On $\gamma_{2}^{+}$we have $u=\pi_{2}^{+} u=1$, and on $\gamma_{2}^{-}$we have $u=\pi_{2}^{+} u=(-1)^{k+1}$. Since $\frac{\partial r_{1}}{\partial x_{1}}$ is a polynomial of degree at most $k-1$ in $x_{1}$, we obtain

$$
\int_{K}\left(u-\Pi^{+} u\right) \frac{\partial r_{1}}{\partial x_{1}} d x_{1} d x_{2}=\int_{K}\left(u-\pi_{1}^{+} u\right) \frac{\partial r_{1}}{\partial x_{1}} d x_{1} d x_{2}=0 .
$$

Thus, $Z_{K, 1}\left(r_{1}, u\right)=0$ for $u\left(x_{1}, x_{2}\right)=x_{1}^{k+1}$.

In the case $u\left(x_{1}, x_{2}\right)=x_{2}^{k+1}$, we integrate by parts and obtain

$$
\begin{gathered}
\int_{K}\left(u-\Pi^{+} u\right) \frac{\partial r_{1}}{\partial x_{1}} d x_{1} d x_{2}=-\int_{K} \frac{\partial\left(u-\Pi^{+} u\right)}{\partial x_{1}} r_{1} d x_{1} d x_{2} \\
+\int_{\gamma_{2}^{+}}\left(u-\pi_{2}^{+} u\right) r_{1} d x_{1}-\int_{\gamma_{2}^{-}}\left(u-\Pi^{+} u\right) r_{1} d x_{2} .
\end{gathered}
$$

Since $\frac{\partial\left(u-\Pi^{+} u\right)}{\partial x_{1}}=0$ and $\Pi^{+} u_{\gamma_{\gamma_{2}^{-}}}=\pi_{2}^{+} u_{\gamma_{\gamma_{2}^{-}}}$due to the special form of $u$, we conclude that $Z_{K, 1}\left(r_{1}, u\right)=0$ also for $u\left(x_{1}, x_{2}\right)=x_{2}^{k+1}$. This completes the proof of (3.6).

For fixed $r_{1} \in \mathcal{Q}^{k}(K)$, the linear functional $u \mapsto Z_{K, 1}\left(r_{1}, u\right)$ is continuous on $H^{s+2}(K)$ with norm bounded by $C\left\|r_{1}\right\|_{0, K}$. Due to (3.6), it vanishes over $\mathcal{P}^{s+1}(K)$ for $0 \leq s \leq$ $k$. Hence, by applying Bramble-Hilbert's Lemma (see [6, Lemma 6], for instance), we obtain for $u \in H^{s+2}(K)$ that

$$
\left|Z_{K, 1}\left(r_{1}, u\right)\right| \leq C|u|_{s+2, K}\left\|r_{1}\right\|_{0, K} .
$$

This proves the assertion on the reference element $(-1,1)^{2}$. The general case follows from a standard scaling argument. 
3.5. Proof of Theorem 2.1. If the exact solution of our model problem, $(\boldsymbol{q}, u)$, belongs to $H^{k+1}(\Omega)^{2} \times H^{k+2}(\Omega)$, with $k \geq 0$, Lemmas 3.4 and 3.6 give

$$
K_{\mathcal{A}}(\boldsymbol{q}, u ; \boldsymbol{q}, u) \leq h^{2 k+1+\alpha}\|u\|_{k+2}^{2}
$$

and

$$
\begin{aligned}
K_{\mathcal{B}}(\boldsymbol{q}, u) \leq & h^{k+\frac{1}{2}-\frac{|\alpha|}{2}}\|u\|_{k+2}+h^{k+\frac{1}{2}}\left\|u_{\Gamma}\right\|_{k+\frac{3}{2}, \Gamma} \\
& +\left|\mathcal{E}_{\mathcal{N}} \cap \mathcal{E}^{-}\right|^{\frac{1}{2}} h^{\min \{s, k\}+\frac{1}{2}}\|u\|_{W^{s+1, \infty}\left(E_{\mathcal{N}}\right)},
\end{aligned}
$$

with $u_{\Gamma}=u_{\left.\right|_{\Gamma}}$. The estimate of the error $\left|\left(\boldsymbol{q}-\boldsymbol{q}_{N}, u-u_{N}\right)\right|_{\mathcal{A}}$ follows now from Lemma 3.1. Notice that $\alpha=0$ gives the best order of convergence in $h$ equal to $k+\frac{1}{2}$. Our assumptions on the domain imply that the solution $\varphi$ of the adjoint problem in Lemma 3.1 belongs to $H^{2}(\Omega)$ and that we have $\|\varphi\|_{2} \leq C\|\lambda\|_{0}$; see $[8,9]$. Hence, we conclude that

$$
\begin{gathered}
K_{\mathcal{A}}(\boldsymbol{q}, u ; \boldsymbol{\Phi}, \varphi) \leq C \begin{cases}h^{k+1}\|u\|_{k+2}\|\lambda\|_{0} & k \geq 1, \\
h^{1+\min (0, \alpha)}\|u\|_{2}\|\lambda\|_{0} & k=0,\end{cases} \\
K_{\mathcal{B}}(\Phi, \varphi) \leq C h^{\frac{1}{2}-\frac{|\alpha|}{2}\|\lambda\|_{0} .}
\end{gathered}
$$

The estimate of $\left\|u-u_{N}\right\|_{0}$ thus follows from Lemma 3.1. Notice that $\alpha=0$ gives again the best order of convergence in $h$ which is $k+1$.

4. Numerical Experiments. In this section, we display a series of numerical experiments showing the computed orders of convergence of the LDG method; we show (i) that the orders given by our theoretical results are sharp, (ii) that they can deteriorate when the stabilization parameter $C_{11}$ is not of order one, (iii) that the exact capture of the boundary conditions induces an unexpected increase of $\frac{1}{2}$ in the order of convergence of the gradient, and (iv) that the orders of convergence are independent of the dimension.

In all experiments, we estimate the orders of convergence of the LDG method as follows. We consider successively refined Cartesian grids $\mathcal{T}_{\ell}, \ell \geq 0$, consisting of $2^{d \ell}$ uniform $d$-dimensional cubes with corresponding mesh size $2^{-\ell+1}$; we present results in two and three space dimensions. If $e\left(\mathcal{T}_{\ell}\right)$ denotes the error on the $\ell$-th mesh, then the numerical order of convergence is computed as follows:

$$
\log \left(\frac{e\left(\mathcal{T}_{\ell}\right)}{e\left(\mathcal{T}_{\ell-1}\right)}\right) / \log (0.5), \quad \ell \geq 1
$$

The results have been obtained with the object-oriented C++ library deal. II developed by Bangerth and Kanschat [1].

4.1. The sharpness of the orders of convergence of Theorem 2.1. We consider the two-dimensional model problem (1.1) on the square $\Omega=(-1,1)^{2}$ with $f$ and boundary conditions chosen in such a way that the exact solution is given by $u\left(x_{1}, x_{2}\right)=\exp \left(x_{1} x_{2}\right)$. We consider two cases: In the first, we impose inhomogeneous Dirichlet boundary conditions on the whole boundary, and in the second, we also impose inhomogeneous Neumann boundary conditions on the edge $\{-1\} \times(-1,1)$. The results are contained in Tables 4.1 and 4.2 where the numerical orders of convergence in the $\mathrm{L}^{2}$ - and $\mathrm{L}^{\infty}$-norm in $u, q_{1}$ and $q_{2}$ of the LDG method with $\mathcal{Q}^{k}$ elements for $k=0, \ldots, 3$ are shown. We take $C_{11}=1.0$ and the coefficients $\boldsymbol{C}_{12}$ as in (2.11) with $\boldsymbol{v}=(1,1)$. 
In Table 4.1, we report the results for Dirichlet boundary conditions imposed on the whole boundary. Note that, because of the symmetry of the problem, the orders of convergence are exactly the same for $q_{1}$ and $q_{2}$. For $k=0$, we see the optimal order of convergence of 1 in the $\mathrm{L}^{2}$-norm of the error of both $u$ and $\boldsymbol{q}$; note that Theorem 2.1 predicts an order of convergence of $\frac{1}{2}$ only for $\boldsymbol{q}$. However, for $k \geq 1$ the $\mathrm{L}^{2}$-rates are of order $k+1$ in $u$ and $k+\frac{1}{2}$ in $\boldsymbol{q}$, in full agreement with Theorem 2.1. The orders on convergence in the $L^{\infty}$-norm of the error in $u$ and $\boldsymbol{q}$ appear to be $k+1$ and $k$, respectively.

The results displayed in Table 4.2 are those for the case of inhomogeneous Neumann boundary conditions on part of the boundary. We see that the orders of convergence in this case are the same as the ones in the previous case.

Thus, the above experiments show that the orders of convergence given by Theorem 2.1 are sharp.

TABLE 4.1

Orders of convergence for the $L D G$ method with $C_{11}=1.0: \Gamma_{\mathcal{N}}=\emptyset$.

\begin{tabular}{|c|c||c|c||c|c|}
\hline & \multicolumn{1}{|c||}{} & \multicolumn{2}{c||}{$u$} & \multicolumn{2}{c|}{$q_{1}$ and $q_{2}$} \\
\hline element & $\ell$ & $\mathrm{L}^{2}$ & $\mathrm{~L}^{\infty}$ & $\mathrm{L}^{2}$ & $\mathrm{~L}^{\infty}$ \\
\hline \multirow{4}{*}{$\mathcal{Q}^{0}$} & 1 & 0.5043 & 0.1284 & 0.7401 & 0.3036 \\
& 2 & 0.7974 & 0.3682 & 0.7257 & 0.4175 \\
& 3 & 0.9975 & 0.6029 & 0.7892 & 0.4574 \\
& 4 & 1.0095 & 0.7873 & 0.8803 & 0.1365 \\
& 5 & 0.9736 & 0.9019 & 0.9398 & 0.2937 \\
& 6 & 0.9683 & 0.9624 & 0.9724 & 0.3856 \\
\hline \multirow{4}{*}{$\mathcal{Q}^{1}$} & 1 & 1.7570 & 0.9753 & 1.5354 & 1.3116 \\
& 2 & 1.7999 & 1.3976 & 1.2669 & 0.7766 \\
& 3 & 1.8496 & 1.6995 & 1.2857 & 0.7150 \\
& 4 & 1.8941 & 1.8549 & 1.3640 & 0.8670 \\
& 5 & 1.9390 & 1.9305 & 1.4251 & 0.9422 \\
& 6 & 1.9681 & 1.8971 & 1.4610 & 0.9747 \\
\hline \multirow{4}{*}{$\mathcal{Q}^{2}$} & 1 & 2.7300 & 1.9615 & 2.3151 & 1.5921 \\
& 2 & 2.8570 & 2.5254 & 2.3071 & 1.6811 \\
& 3 & 2.8676 & 2.7695 & 2.3280 & 1.8300 \\
& 4 & 2.8999 & 2.8862 & 2.3901 & 1.8988 \\
& 5 & 2.9382 & 2.9428 & 2.4387 & 1.9392 \\
& 6 & 2.9661 & 2.8316 & 2.4678 & 1.9658 \\
\hline \multirow{6}{*}{$\mathcal{Q}^{3}$} & 1 & 3.6933 & 2.9236 & 3.1730 & 1.8719 \\
& 2 & 3.8108 & 3.5453 & 3.2270 & 2.5280 \\
& 3 & 3.8660 & 3.7719 & 3.3094 & 2.7551 \\
& 4 & 3.9120 & 3.8827 & 3.3859 & 2.8770 \\
& 5 & 3.9490 & 3.9398 & 3.4380 & 2.9415 \\
& 6 & 3.9661 & 3.8249 & 3.4676 & 2.9724 \\
\hline
\end{tabular}

4.2. The effect of the choice of $C_{11}$. Next, we test the effect of the choice of the coefficients $C_{11}$ on the orders of convergence of the LDG method. We consider the same problem as in the previous experiments, case $\Gamma_{\mathcal{N}}=\emptyset$, and use $\mathcal{Q}^{1}$ and $\mathcal{Q}^{2}$ elements. We only show the numerical orders of convergence for the finest grids. 
TABLE 4.2

Orders of convergence of the $L D G$ method with $C_{11}=1.0: \Gamma_{\mathcal{N}} \neq \emptyset$.

\begin{tabular}{|c|c|c|c|c|c|c|c|}
\hline & & \multicolumn{2}{|c|}{$u$} & \multicolumn{2}{|c|}{$q_{1}$} & \multicolumn{2}{|c|}{$q_{2}$} \\
\hline element & $\ell$ & $\mathrm{L}^{2}$ & $\mathrm{~L}^{\infty}$ & $\mathrm{L}^{2}$ & $\mathrm{~L}^{\infty}$ & $\mathrm{L}^{2}$ & $L^{\infty}$ \\
\hline \multirow{6}{*}{$\mathcal{Q}^{0}$} & 1 & 0.4279 & 0.0833 & 0.5632 & 0.1687 & 0.7419 & 0.2817 \\
\hline & 2 & 0.7818 & 0.3935 & 0.6860 & 0.2553 & 0.7330 & 0.4255 \\
\hline & 3 & 0.9281 & 0.6447 & 0.9021 & 0.4920 & 0.8168 & 0.5801 \\
\hline & 4 & 0.9589 & 0.8134 & 1.0231 & 0.7055 & 0.9221 & 0.2833 \\
\hline & 5 & 0.9687 & 0.9083 & 1.0463 & 0.8479 & 0.9793 & 0.4939 \\
\hline & 6 & 0.9795 & 0.9555 & 1.0303 & 0.9283 & 0.9954 & 0.6270 \\
\hline \multirow{6}{*}{$\mathcal{Q}^{1}$} & 1 & 1.5640 & 0.8492 & 1.4414 & 0.8857 & 1.4867 & 1.0768 \\
\hline & 2 & 1.7767 & 1.4022 & 1.4194 & 1.1787 & 1.3096 & 1.1126 \\
\hline & 3 & 1.8567 & 1.6995 & 1.4695 & 1.1444 & 1.3162 & 0.7155 \\
\hline & 4 & 1.9167 & 1.8549 & 1.5024 & 0.8670 & 1.3843 & 0.8669 \\
\hline & 5 & 1.9559 & 1.9305 & 1.5117 & 0.9422 & 1.4379 & 0.9422 \\
\hline & 6 & 1.9777 & 1.8971 & 1.5097 & 0.9747 & 1.4683 & 0.9746 \\
\hline \multirow{6}{*}{$\mathcal{Q}^{2}$} & 1 & 2.6095 & 1.8429 & 2.4038 & 1.8936 & 2.3275 & 1.8386 \\
\hline & 2 & 2.8329 & 2.5220 & 2.4372 & 2.1265 & 2.3217 & 1.8231 \\
\hline & 3 & 2.8806 & 2.7695 & 2.4719 & 1.9471 & 2.3803 & 1.8300 \\
\hline & 4 & 2.9230 & 2.8862 & 2.4963 & 1.8988 & 2.4320 & 1.8988 \\
\hline & 5 & 2.9563 & 2.9428 & 2.5042 & 1.9392 & 2.4631 & 1.9392 \\
\hline & 6 & 2.9770 & 2.8316 & 2.5044 & 1.9658 & 2.4806 & 1.9658 \\
\hline \multirow{6}{*}{$\mathcal{Q}^{3}$} & 1 & 3.6125 & 2.8600 & 3.3206 & 2.7792 & 3.1634 & 2.5820 \\
\hline & 2 & 3.8202 & 3.5475 & 3.3839 & 2.9180 & 3.2859 & 2.5593 \\
\hline & 3 & 3.8916 & 3.7719 & 3.4665 & 2.7551 & 3.3743 & 2.7551 \\
\hline & 4 & 3.9375 & 3.8827 & 3.4950 & 2.8770 & 3.4318 & 2.8770 \\
\hline & 5 & 3.9664 & 3.9398 & 3.5022 & 2.9414 & 3.4643 & 2.9414 \\
\hline & 6 & 3.9805 & 3.8264 & 3.5024 & 2.9722 & 3.4815 & 2.9721 \\
\hline
\end{tabular}

The results are displayed in Tables 4.3 and 4.4. We must compare all these results with those with $C_{11}=1$ obtained in the first set of experiments. We see that when $C_{11}$ is of order $h^{-1}$, the order of convergence in $u$ remains $k+1$ but the order of convergence in $\boldsymbol{q}$ degrades from $k+\frac{1}{2}$ to only $k$, as predicted by our analysis; see section 3.5.

We also see that taking $C_{11}=h^{-1}$ at the outflow boundary and $C_{11}$ of order one elsewhere only results in a slight reduction of the $\mathrm{L}^{\infty}$-orders of convergence.

In the remaining cases, we take $C_{11}$ to be of order $h$ in all the domain and then in all but the outflow boundary where it is taken to be of order $h^{-1}$. We observe a slight degradation of all the orders of convergence.

These results indicate that the best choice of the stabilization parameter $C_{11}$ for the LDG method is to take it of order one, as predicted by our analysis.

4.3. Piecewise polynomial boundary conditions. The purpose of these numerical experiments is to show that if the boundary data are piecewise polynomials of degree $k$, the order of convergence of the $\mathrm{L}^{2}$-norm of the error in $\boldsymbol{q}$ is optimal, that is, $k+1$, and not only $k+\frac{1}{2}$ as predicted by Theorem 2.1 and shown to be sharp in sub-section 4.1. 
TABLE 4.3

Orders of convergence of the $L D G$ method with $\mathcal{Q}^{1}$ elements.

\begin{tabular}{|c|c||c|c||c|c|}
\hline \multicolumn{1}{|c||}{} & \multicolumn{1}{c||}{} & \multicolumn{2}{c||}{$u$} & \multicolumn{2}{c|}{$q_{1}$ and $q_{2}$} \\
\hline$C_{11}$ & $\ell$ & $\mathrm{L}^{2}$ & $\mathrm{~L}^{\infty}$ & $\mathrm{L}^{2}$ & $\mathrm{~L}^{\infty}$ \\
\hline $1 / h$ & 5 & 1.9607 & 1.9550 & 1.1409 & 0.8816 \\
& 6 & 1.9792 & 1.9057 & 1.1019 & 0.9366 \\
\hline $1 / h$ on $\mathcal{E}^{+}$ & 5 & 1.9331 & 1.7799 & 1.4240 & 0.8619 \\
1.0 elsewhere & 6 & 1.9646 & 1.7914 & 1.4605 & 0.9268 \\
\hline $\mathrm{h}$ & 5 & 1.8916 & 1.8810 & 1.4167 & 0.9405 \\
& 6 & 1.8603 & 1.7887 & 1.4564 & 0.9701 \\
\hline $1 / h$ on $\mathcal{E}^{+}$ & 5 & 1.8837 & 1.8810 & 1.4157 & 0.8698 \\
$h$ elsewhere & 6 & 1.8563 & 1.7887 & 1.4556 & 0.9319 \\
\hline
\end{tabular}

TABLE 4.4

Orders of convergence of the LDG method with $\mathcal{Q}^{2}$ elements.

\begin{tabular}{|c|c||c|c||c|c|}
\hline \multicolumn{1}{|c|}{} & \multicolumn{1}{|c||}{} & \multicolumn{2}{c||}{$u$} & \multicolumn{2}{c|}{$q_{1}$ and $q_{2}$} \\
\hline$C_{11}$ & $\ell$ & $\mathrm{L}^{2}$ & $\mathrm{~L}^{\infty}$ & $\mathrm{L}^{2}$ & $\mathrm{~L}^{\infty}$ \\
\hline $1 / h$ & 5 & 2.9555 & 2.9541 & 2.2223 & 1.8475 \\
& 6 & 2.9754 & 2.9584 & 2.1685 & 1.9228 \\
\hline $1 / h$ on $\mathcal{E}^{+}$ & 5 & 2.9340 & 2.8836 & 2.4358 & 1.8828 \\
1.0 elsewhere & 6 & 2.9634 & 2.7424 & 2.4663 & 1.9427 \\
\hline h & 5 & 2.8559 & 2.9524 & 2.4350 & 1.9483 \\
& 6 & 2.8240 & 2.5482 & 2.4656 & 1.9742 \\
\hline $1 / h$ on $\mathcal{E}^{+}$ & 5 & 2.8505 & 2.8642 & 2.4325 & 1.8760 \\
$h$ elsewhere & 6 & 2.8211 & 2.4554 & 2.4643 & 1.9365 \\
\hline
\end{tabular}

We consider two test problems. In the first, we take homogeneous Dirichlet boundary conditions and $f$ such that the exact solution is $u\left(x_{1}, x_{2}\right)=\cos \left(\frac{\pi}{2} x_{1}\right) \cos \left(\frac{\pi}{2} x_{2}\right)$. In the second, we take piecewise quadratic Dirichlet boundary conditions and $f$ such that the exact solution is $u\left(x_{1}, x_{2}\right)=x_{1}^{2}+x_{2}^{2}+\cos \left(\frac{\pi}{2} x_{1}\right) \cos \left(\frac{\pi}{2} x_{2}\right)$.

The results of the first problem are reported in Table 4.5 where we can see that the optimal order of convergence of $k+1$ for the $\mathrm{L}^{2}$ - and $\mathrm{L}^{\infty}$-norms of the errors in both $u$ and $\boldsymbol{q}$ are obtained; the results for $k=0,1,2,3$ are displayed.

The results of the second problem are reported in Table 4.6, where we see that the optimal order of convergence of $k+1$ for the $\mathrm{L}^{2}$ - and $\mathrm{L}^{\infty}$-norms of the errors in both $u$ and $\boldsymbol{q}$ are obtained for $k \geq 2$, as claimed. For $k<2$, the order of convergence in the $\mathrm{L}^{2}$-norm of the error in $\boldsymbol{q}$ is $k+\frac{1}{2}$ only which nothing but the order of convergence predicted by Theorem 2.1 .

To better understand this phenomenon, we plot the errors in $q_{1}$ for $\mathcal{Q}^{1}$ and $\mathcal{Q}^{2}$ elements in Figs. 4.1 and 4.2, respectively; the triangulation has $16 \times 16$ elements and corresponds to the index $\ell=4$. We immediately see the oscillatory behavior of the error typical of finite element methods. In Fig. 4.1, we see that the error obtained with $\mathcal{Q}^{1}$ elements is bigger at the boundary than at the interior. This, together with the fact that the order of convergence in $\mathrm{L}^{2}$ is $\frac{3}{2}$ whereas the order of convergence in $\mathrm{L}^{\infty}$ is only 1 , suggests that the error at the boundary is a factor of order $h^{-\frac{1}{2}}$ bigger 
than the error at the interior of the domain. On the other hand, the behavior of the error with $\mathcal{Q}^{2}$ elements is rather different, as can be seen in Fig. 4.2. Indeed, the error behaves in the same way at the boundary and at the interior; this is further confirmed by the fact that both the the order of convergence in $\mathrm{L}^{2}$ and the one in $\mathrm{L}^{\infty}$ are equal to $k+1$.

These experiments justify our contention that the optimal order of convergence in $\boldsymbol{q}$ can be reached if the boundary conditions are piecewise polynomials of degree $k$. Our theoretical analysis does not explain this phenomenon.

TABLE 4.5

Orders of convergence for the $L D G$ method with $C_{11}=1.0: g_{\mathcal{D}}=0, \Gamma_{\mathcal{N}}=\emptyset$.

\begin{tabular}{|c|c||c|c||c|c|}
\hline & \multicolumn{1}{|c||}{} & \multicolumn{2}{c||}{$u$} & \multicolumn{2}{c|}{$q_{1}$ and $q_{2}$} \\
\hline element & $\ell$ & $\mathrm{L}^{2}$ & $\mathrm{~L}^{\infty}$ & $\mathrm{L}^{2}$ & $\mathrm{~L}^{\infty}$ \\
\hline $\mathcal{Q}^{0}$ & 5 & 0.8913 & 0.9278 & 0.9299 & 0.8973 \\
& 6 & 0.9456 & 0.9658 & 0.9662 & 0.9483 \\
\hline $\mathcal{Q}^{1}$ & 5 & 2.0352 & 1.9750 & 2.0000 & 1.9748 \\
& 6 & 2.0213 & 1.9878 & 2.0003 & 1.9858 \\
\hline $\mathcal{Q}^{2}$ & 5 & 2.9637 & 3.0266 & 2.9689 & 3.0292 \\
& 6 & 2.9815 & 3.0150 & 2.9855 & 3.0161 \\
\hline $\mathcal{Q}^{3}$ & 5 & 4.0435 & 3.9806 & 4.0087 & 3.9771 \\
& 6 & 4.0247 & 3.9918 & 4.0041 & 3.9748 \\
\hline
\end{tabular}

TABLE 4.6

Orders of convergence for the $L D G$ method with $C_{11}=1.0: g_{\mathcal{D}}$ quadratic, $\Gamma_{\mathcal{N}}=\emptyset$.

\begin{tabular}{|c|c||c|c||c|c|}
\hline \multicolumn{1}{|c||}{} & & \multicolumn{2}{c||}{$u$} & \multicolumn{2}{c|}{$q_{1}$ and $q_{2}$} \\
\hline element & $\ell$ & $\mathrm{L}^{2}$ & $\mathrm{~L}^{\infty}$ & $\mathrm{L}^{2}$ & $\mathrm{~L}^{\infty}$ \\
\hline $\mathcal{Q}^{0}$ & 5 & 0.9886 & 0.9739 & 0.7359 & 0.0052 \\
& 6 & 0.9935 & 1.0066 & 0.8009 & 0.0142 \\
\hline $\mathcal{Q}^{1}$ & 5 & 2.0030 & 1.9552 & 1.4906 & 1.0160 \\
& 6 & 2.0015 & 1.9775 & 1.4976 & 1.0091 \\
\hline $\mathcal{Q}^{2}$ & 5 & 2.9637 & 3.0266 & 2.9689 & 3.0292 \\
& 6 & 2.9815 & 3.0150 & 2.9855 & 3.0162 \\
\hline $\mathcal{Q}^{3}$ & 5 & 4.0435 & 3.9804 & 4.0087 & 3.9762 \\
& 6 & 4.0245 & 3.9909 & 4.0036 & 3.9717 \\
\hline
\end{tabular}




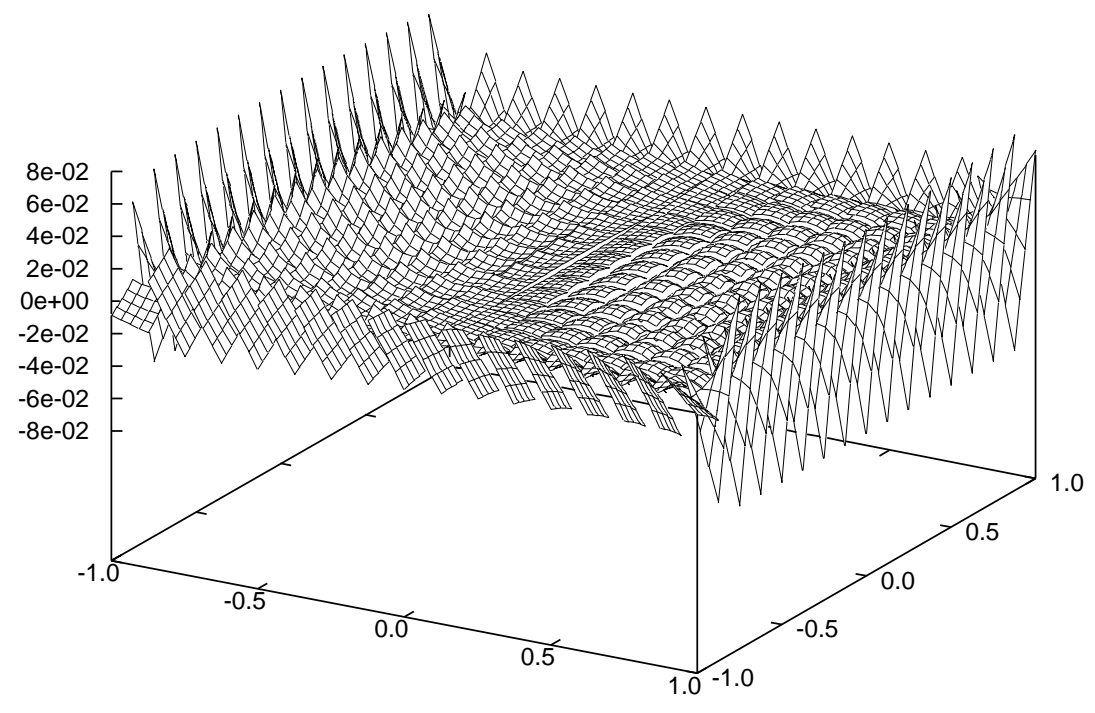

FIG. 4.1. The error in the first component of the gradient for $\mathcal{Q}^{1}: g_{\mathcal{D}}$ quadratic, $\Gamma_{\mathcal{N}}=\emptyset$.

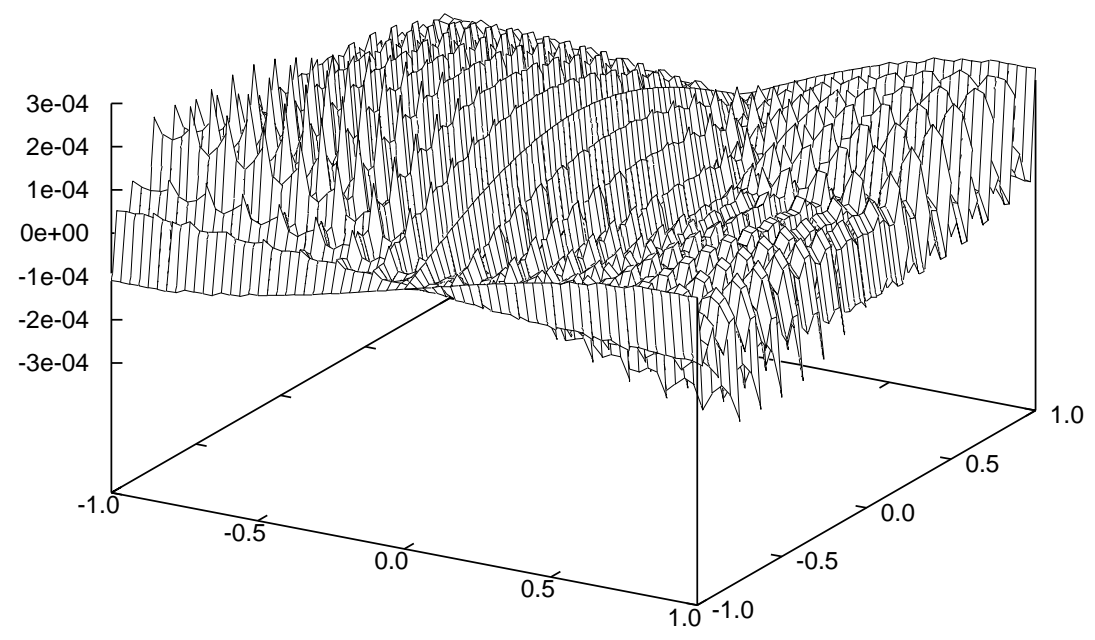

FIG. 4.2. The error in the first component of the gradient for $\mathcal{Q}^{2}: g_{\mathcal{D}}$ quadratic, $\Gamma_{\mathcal{N}}=\emptyset$. 
4.4. A three-dimensional example. In this experiment, we consider the model problem (1.1) on the three-dimensional domain $\Omega=(-1,1)^{3}$. We take Dirichlet boundary conditions and $f$ such that the exact solution is

$$
u\left(x_{1}, x_{2}, x_{3}\right)=x_{1}^{2}+x_{2}^{2}+x_{3}^{2}+\cos \left(\frac{\pi}{2} x_{1}\right) \cos \left(\frac{\pi}{2} x_{2}\right) \cos \left(\frac{\pi}{2} x_{3}\right) .
$$

The results are displayed in Table 4.7; the computation on level 5 with $\mathcal{Q}^{2}$ did not fit into the computers available to us. We can see that the orders of convergence are similar to those obtained in the corresponding two-dimensional test problem in the previous sub-section, cf. Table 4.6. This gives an indication that the orders of convergence of the LDG method in three space dimension behave in the same way they do in the two-dimensional case.

TABLE 4.7

Orders of convergence for the $L D G$ method with $C_{11}=1.0$ in $3 D: g_{\mathcal{D}}$ quadratic, $\Gamma_{\mathcal{N}}=\emptyset$.

\begin{tabular}{|c|c||c||c|}
\hline & & $u$ & $q_{1}$ and $q_{2}$ \\
\hline element & $\ell$ & $\mathrm{L}^{2}$ & $\mathrm{~L}^{2}$ \\
\hline \multirow{3}{*}{$\mathcal{Q}^{0}$} & 3 & 0.9389 & 0.5118 \\
& 4 & 0.9367 & 0.6177 \\
& 5 & 0.9452 & 0.7203 \\
\hline \multirow{3}{*}{$\mathcal{Q}^{1}$} & 3 & 1.8573 & 1.3374 \\
& 4 & 1.9278 & 1.4345 \\
& 5 & 1.9636 & 1.4723 \\
\hline \multirow{3}{*}{$\mathcal{Q}^{2}$} & 3 & 2.9204 & 2.8642 \\
& 4 & 2.9326 & 2.9338 \\
& 5 & n/a & n/a \\
\hline
\end{tabular}

5. Concluding remarks. In this paper we have shown that the LDG method on Cartesian grids and with a special numerical flux super-converges; the proof of this result is based on suitable defined projections $\Pi$ and $\Pi$ exhibiting a tensor product structure. This work extends the corresponding result by LeSaint and Raviart [10] for the DG method for linear hyperbolic problems and that by Castillo [2] and Castillo, Cockburn, Schötzau and Schwab [4] for the LDG method applied to the one-dimensional transient convection-diffusion. Extensions of this work to more general elliptic and both steady and transient convection-diffusion problems can easily be made.

\section{REFERENCES}

[1] W. Bangerth and G. Kanschat, Concepts for object-oriented finite element software - the deal.II library, Preprint 99-43, Sonderforschungbereich 3-59, IWR, Universität Heidelberg, October 1999.

[2] P. Castillo, An optimal error estimate for the local discontinuous Galerkin method, First International Symposium on Discontinuous Galerkin Methods (B. Cockburn, G.E. Karniadakis, and C.-W. Shu, eds.), Lecture Notes in Computational Science and Engineering, vol. 11, Springer Verlag, February 2000, pp. 285-290.

[3] P. Castillo, B. Cockburn, I. Perugia, and D. Schötzau, An a priori error analysis of the local discontinuous Galerkin method for elliptic problems, 00 (0000).

[4] P. Castillo, B. Cockburn, D. Schötzau, and Ch. Schwab, An optimal a priori error estimate for the hp-version of the local discontinuous Galerkin method for convection-diffusion problems, IMA Research Report 1689, University of Minnesota, 2000, submitted. 
[5] P. Ciarlet, The finite element method for elliptic problems, North Holland, 1978.

[6] P.G. Ciarlet and P.A. Raviart, General Lagrange and Hermite interpolation in $R^{n}$ with applications to finite element methods, Arch. Rat. Mech. Anal. 46 (1972), 177-199.

[7] B. Cockburn and C.W. Shu, The local discontinuous Galerkin finite element method for convection-diffusion systems, SIAM J. Numer. Anal. 35 (1998), 2440-2463.

[8] P. Grisvard, Elliptic problems in nonsmooth domains, Monographs and Studies in Mathematics, 34, Pitman, Boston, 1985.

[9] , Singularities in boundary value problems, Research Notes in Applied Mathematics, Masson, Paris, 1992.

[10] P. LeSaint and P.A. Raviart, On a finite element method for solving the neutron transport equation, Mathematical aspects of finite elements in partial differential equations (C. de Boor, ed.), Academic Press, 1974, pp. 89-145. 